\title{
A dual of the rectangle-segmentation problem for binary matrices
}

\author{
Thomas Kalinowski \\ Institut für Mathematik, Universität Rostock, 18051 Rostock, Germany \\ thomas.kalinowski@uni-rostock.de
}

Submitted: Apr 23, 2009; Accepted: Jul 11, 2009; Published: Jul 24, 2009

Mathematics Subject Classification: 90C27, 90C46

\begin{abstract}
We consider the problem to decompose a binary matrix into a small number of binary matrices whose 1-entries form a rectangle. We show that the linear relaxation of this problem has an optimal integral solution corresponding to a well known geometric result on the decomposition of rectilinear polygons.
\end{abstract}

\section{Introduction}

In the context of intensity modulated radiation therapy several decomposition problems for nonnegative integer matrices have been considered. One of these is the decomposition into a small number of binary matrices whose 1-entries form a rectangle. There is an example showing that in general the linear relaxation of this problem has no optimal integral solution [1]. On the other hand, the same paper contains an algorithm based on the revised simplex method that uses only very few Gomory cuts. In computational experiments, this algorithm provided exact solutions for matrices of reasonable size in short time.

In the present paper we consider the special case that the input matrix is already binary: $A \in\{0,1\}^{m \times n}$. In this case the integer optimization problem is equivalent to a well studied geometric problem: the decomposition of a rectilinear polygon into the minimal number of rectangles. Our main result is that the minimal number of rectangles in such a decomposition equals the optimal objective in the relaxed matrix decomposition problem. In other words, the integrality gap vanishes, provided the input matrix is binary. This solves problem 1 of [1].

\section{Notation and problem formulation}

Let $A$ be a binary matrix of size $m \times n$. A rectangle matrix is an $m \times n$-matrix $S=\left(s_{i j}\right)$ such that for some integers $k_{1}, k_{2}, l_{1}$ and $l_{2}$ with $1 \leq k_{1} \leq k_{2} \leq m$ and $1 \leq l_{1} \leq l_{2} \leq n$, 
we have

$$
s_{i j}= \begin{cases}1 & \text { if } k_{1} \leq i \leq k_{2} \text { and } l_{1} \leq j \leq l_{2}, \\ 0 & \text { otherwise }\end{cases}
$$

The rectangle segmentation problem is the following:

RSP. Find a decomposition $A=S_{1}+\cdots+S_{t}$ with rectangle matrices $S_{1}, \ldots, S_{t}$ such that $t$ is minimal.

A linear relaxation of this problem is the following.

RSP-Relax. Find a linear combination $A=x_{1} S_{1}+\cdots+x_{t} S_{t}$ with rectangle matrices $S_{1}, \ldots, S_{t}$ and $0 \leq x_{i} \leq 1$ for $i=1, \ldots, t$ such that $x_{1}+\cdots+x_{t}$ is minimal.

The integral problem $\mathbf{R S P}$ can be formulated in a geometric setup as follows. We associate $A$ with a rectangular $m \times n$-array of unit squares in the plane. The set $P=\left\{(i, j): a_{i j}=\right.$ $1\}$ corresponds to a rectilinear polygon whose boundary consists of line segments with integer coordinates. Clearly, a solution of the problem RSP is precisely the decomposition of $P$ into the minimal number of rectangles. In order to state the solution to the polygon decomposition problem we need some notation. Let $N, c$ and $k$ be the number of vertices, connected components and holes of $P$, respectively. This notation has to be clarified by two remarks (see Fig. 1 for illustrations).

1. If $P$ can be decomposed into two or more polygons which intersect pairwise in isolated vertices these vertices are counted twice and we consider the parts as different connected components.

2. Similarly, if the boundary of a hole intersects the outer boundary or another hole only in isolated vertices these vertices are counted twice.

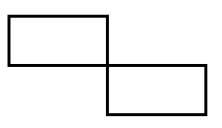

$P_{1}$

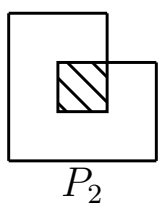

Figure 1: Two polygons. The parameters are $N=8, c=2$ and $k=0$ for $P_{1}$, and $N=10$, $c=1, k=1$ for $P_{2}$.

We call a vertex of $P$ convex if the interior angle at this vertex is $90^{\circ}$ and concave if it is $270^{\circ}$. A chord of $P$ is a line segment that lies completely inside $P$, connects two concave vertices and is parallel to one of the coordinate axes. The chords parallel to the $x$-axis and the $y$-axis are called horizontal and vertical, respectively. We associate a bipartite graph $G=(H \cup V, E)$ with $P$. The vertex sets $H$ and $V$ are the sets of horizontal and vertical chords, respectively, and two chords are connected by an edge if they intersect (Fig. 2). Let $\alpha$ be the maximal cardinality of an independent set in the graph associated to $P$. The following theorem of Lipski et al. (which was reproved by several authors), characterizes the minimal number of rectangles in a decomposition of $P$. 

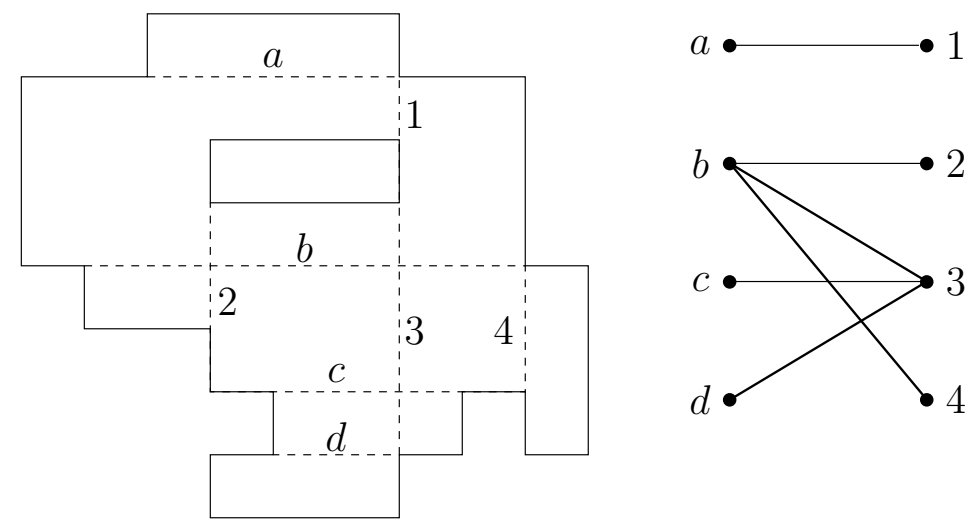

Figure 2: The graph associated to a polygon.

Theorem $1([2,3,4])$. The minimal number of rectangles in a decomposition of $P$ equals

$$
\frac{N}{2}-c+k-\alpha
$$

We want to show that there is no integrality gap in the rectangle segmentation problem for binary matrices. This can be done by proving that the optimal objective value for RSP-Relax is at least $\frac{N}{2}-c+k-\alpha$. In order to do this we will present a feasible solution for the dual problem with exactly this objective value. For a binary matrix $A$, the set of variables in RSP-Relax corresponds to the set of rectangles that are completely contained in $P$. Indexing these rectangles by the numbers $1, \ldots, T$, i.e. $S^{(1)}, \ldots, S^{(T)}$ are precisely the rectangle matrices whose 1-entries are contained in $P$, we can reformulate RSP-Relax as follows.

$$
\begin{array}{rlrl}
\sum_{t=1}^{T} s_{i j}^{(t)} x_{t} & =1 & & \text { for }(i, j) \in P, \\
x_{t} & \geq 0 & & \text { for } t=1, \ldots, T, \\
\sum_{t=1}^{T} x_{t} & \rightarrow \min . &
\end{array}
$$

Dualizing, we obtain the problem RSP-Dual:

$$
\begin{aligned}
\sum_{i=k_{1}}^{k_{2}} \sum_{j=l_{1}}^{l_{2}} y_{i j} & \leq 1 \quad \text { for }\left[k_{1}, k_{2}\right] \times\left[l_{1}, l_{2}\right] \subseteq P, \\
\sum_{(i, j) \in P} y_{i j} & \rightarrow \max .
\end{aligned}
$$

This dual problem has a nice interpretation. The polygon $P$ is considered as a set of unit squares and we want to fill these squares with numbers such that the sum over every rectangle contained in $P$ is bounded by 1 , and the total sum is maximized under this 
constraint. We start with a simple observation which allows us to restrict our attention to dual solutions of a special type. Let $\mathcal{R}$ be the set of rectangles into which $P$ is decomposed when the boundary lines at the concave vertices are extended until they meet the opposite boundary of $P$. For an illustration see Fig. 3, where $|\mathcal{R}|=16$ and, for instance, the big square in the middle is the element $[2,4] \times[4,6] \in \mathcal{R}$. We call the elements of $\mathcal{R}$ basic rectangles. The following lemma asserts that we may assume that only in the upper left corner of a basic rectangle the entry of $\boldsymbol{y}$ is nonzero.

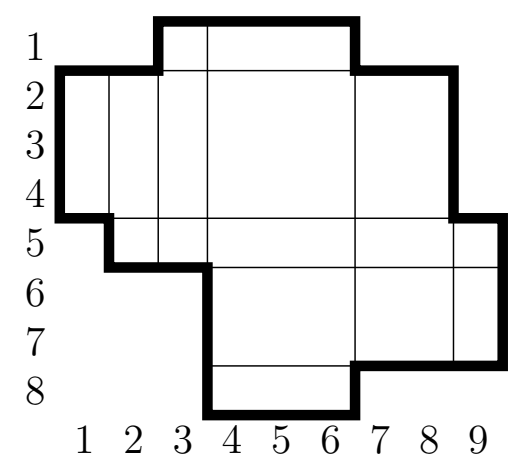

Figure 3: Decomposition of a polygon $P$ into basic rectangles.

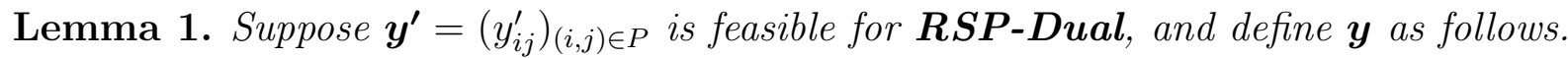
For every $\left[i_{1}, i_{2}\right] \times\left[j_{1}, j_{2}\right] \in \mathcal{R}$ put

$$
y_{i j}= \begin{cases}\sum_{i^{\prime}=i_{1}}^{i_{2}} \sum_{j^{\prime}=j_{1}}^{j_{2}} y_{i^{\prime} j^{\prime}}^{\prime} & \text { for }(i, j)=\left(i_{1}, j_{1}\right), \\ 0 & \text { for }(i, j) \in\left(\left[i_{1}, i_{2}\right] \times\left[j_{1}, j_{2}\right]\right) \backslash\left\{\left(i_{1}, j_{1}\right)\right\} .\end{cases}
$$

Then $\boldsymbol{y}$ is also feasible, and the objective value for $\boldsymbol{y}$ is the same as for $\boldsymbol{y}^{\prime}$.

Proof. Let $R:=\left[k_{1}, k_{2}\right] \times\left[l_{1}, l_{2}\right] \subseteq P$, and let $\mathcal{R}_{0}$ denote the set of basic rectangles having their upper left corner in $R$. More formally,

$$
\mathcal{R}_{0}=\left\{\left[i_{1}, i_{2}\right] \times\left[j_{1}, j_{2}\right] \in \mathcal{R}:\left(i_{1}, j_{1}\right) \in R\right\} .
$$

The union of the elements of $\mathcal{R}_{0}$ is a rectangle $R^{\prime}=\left[k_{1}^{\prime}, k_{2}^{\prime}\right] \times\left[l_{1}^{\prime}, l_{2}^{\prime}\right] \subseteq P$ with

$$
\sum_{i=k_{1}}^{k_{2}} \sum_{j=l_{1}}^{l_{2}} y_{i j}=\sum_{i=k_{1}^{\prime}}^{k_{2}^{\prime}} \sum_{j=l_{1}^{\prime}}^{l_{2}^{\prime}} y_{i j}^{\prime} .
$$

Fig. 4 illustrates the step from $R$ to $R^{\prime}$ for $R=[2,5] \times[3,7]$ and $R^{\prime}=[3,6] \times[3,8]$. Now the feasibility of $\boldsymbol{y}^{\prime}$ implies

$$
\sum_{i=k_{1}}^{k_{2}} \sum_{j=l_{1}}^{l_{2}} y_{i j} \leq 1,
$$

and consequently, the feasibility of $\boldsymbol{y}$. The final statement about the objective values is obvious. 


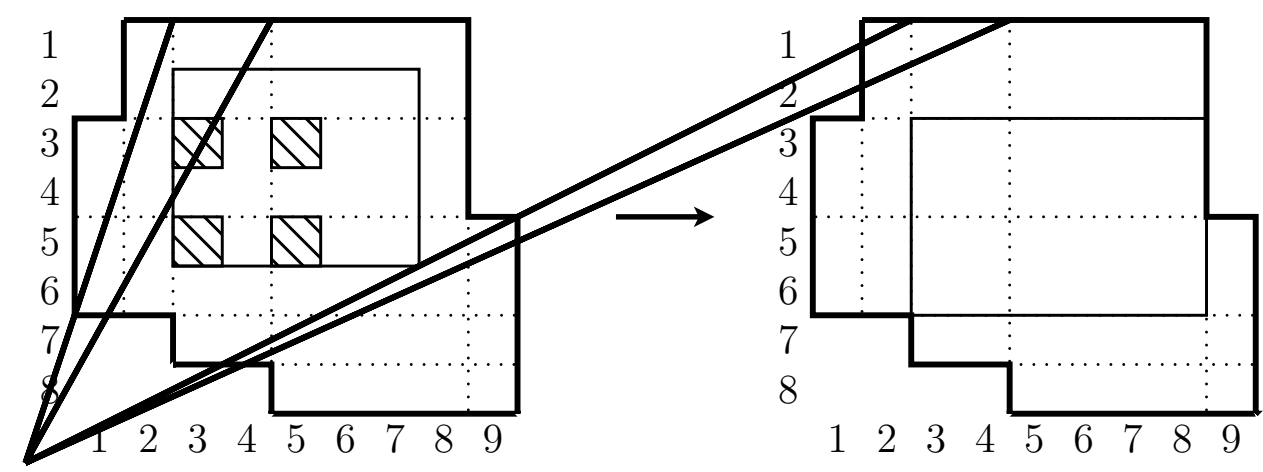

Figure 4: The transition from $R$ to $R^{\prime}$. The upper left corners of the elements of $\mathcal{R}_{0}$ are shaded in the left drawing.

Clearly, a solution $\boldsymbol{y}$ of the form described in Lemma 1 can be identified with the function

$$
g: \mathcal{R} \rightarrow \mathbb{R}, \quad\left[k_{1}, k_{2}\right] \times\left[l_{1}, l_{2}\right] \mapsto y_{k_{1}, l_{1}} .
$$

This is illustrated in Figure 5, where all the values of $g$ are in $\{0, \pm 1\}$. It will turn out that these special values are sufficient to define an optimal solution for RSP-Dual. The

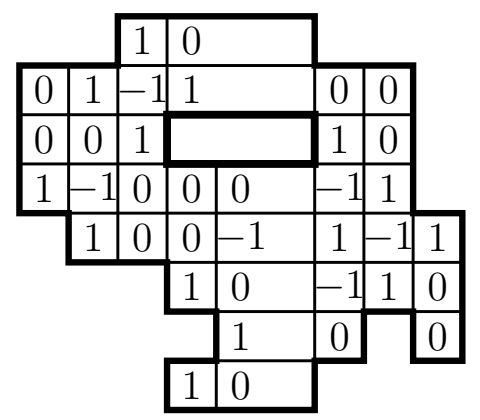

Figure 5: An example solution for RSP-Dual.

same argument as in the proof of Lemma 1 shows that for checking the feasibility of such a function $g$ it is sufficient to consider the constraints for rectangles that are unions of basic rectangles. We call these rectangles essential.

\section{The case $\alpha=0$}

Let us assume $\alpha(G)=0$, i.e. $P$ has no chords at all. We fix an orientation for the lines that are used to decompose $P$ into basic rectangles. The orientation is defined by pointing away from the concave vertices towards the interior of $P$ (see Fig. 6 for an illustration).

We will define the values $g(R)$ for basic rectangles $R$ depending on the orientation of the boundary of $R$. First, we need some additional notation. The vertices of basic rectangles that are either in the interior of $P$ or concave vertices of $P$ are called intersection 


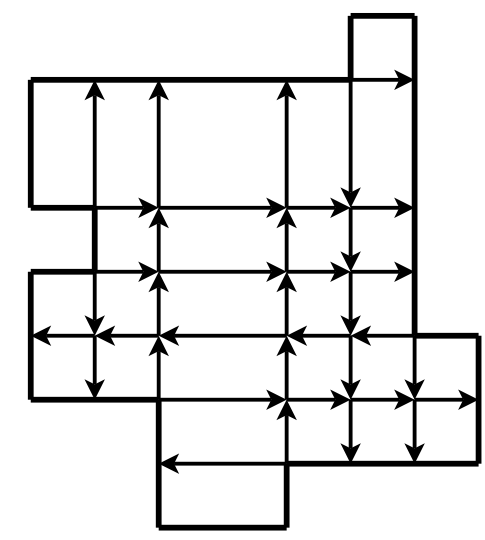

Figure 6: The orientation of the boundaries of the basic rectangles.

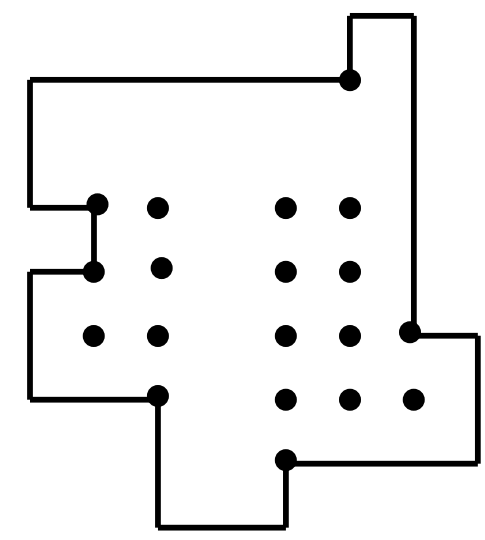

Figure 7: The intersection points.

points. The set of all intersection points is denoted by $I$. In Fig. 7 the intersection points are marked by dots.

Definition. A vertex $a$ of an essential rectangle $\mathcal{A} \subseteq \mathcal{R}$ is called a source (with respect to $\mathcal{A}$ ) if the two line segments on the boundary of $\mathcal{A}$ that start from $a$ are oriented away from $a$. In addition, let $q(\mathcal{A}) \in\{0,1,2\}$ be the number of sources for $\mathcal{A}$.

Now we can define a function $g: \mathcal{R} \rightarrow\{0, \pm 1\}$ which turns out to be an optimal solution for RSP-Dual:

$$
g(R)=1-q(R) \quad(R \in \mathcal{R}) .
$$

In order to show the feasibility of this function we observe that the value extends to essential rectangles.

Lemma 2. For any essential rectangle $\mathcal{A} \subseteq \mathcal{R}$, we have

$$
\sum_{R \in \mathcal{A}} g(R)=1-q(\mathcal{A})
$$

In particular, $g: \mathcal{R} \rightarrow\{0, \pm 1\}$ defines a feasible solution for $\boldsymbol{R S P - D u a l}$.

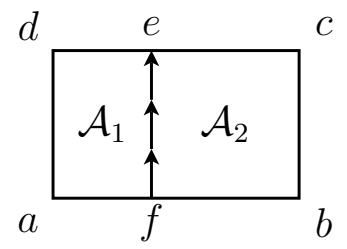

Figure 8: The induction step in the proof of Lemma 2. 
Proof. We proceed by induction on $|\mathcal{A}|$. For $|\mathcal{A}|=1$, the statement is precisely the definition of $g$. For $|\mathcal{A}|>1, \mathcal{A}$ is the union of two rectangles $\mathcal{A}_{1} \cup \mathcal{A}_{2}$ as indicated in Fig. 8. By induction, we have

$$
\sum_{R \in \mathcal{A}_{i}} g(R)=1-q\left(\mathcal{A}_{i}\right) \quad(i \in\{1,2\})
$$

The vertices $a, b, c$ and $d$ are sources for $\mathcal{A}$ iff they are sources for the respective $\mathcal{A}_{i}$. The vertex $e$ is not a source for any of the considered rectangles. Finally, it is easy to see that $f$ is a source in precisely one of the rectangles $\mathcal{A}_{i}$, because it is an intersection point. This yields $q(\mathcal{A})=q\left(\mathcal{A}_{1}\right)+q\left(\mathcal{A}_{2}\right)-1$, hence

$$
\sum_{R \in \mathcal{A}} g(R)=\sum_{R \in \mathcal{A}_{1}} g(R)+\sum_{R \in \mathcal{A}_{2}} g(R)=\left(1-q\left(\mathcal{A}_{1}\right)\right)+\left(1-q\left(\mathcal{A}_{1}\right)\right)=1-q(\mathcal{A})
$$

We observe that every intersection point $a$ is a source for exactly one basic rectangle with vertex $a$. Hence by a simple double counting argument, the objective value for the function $g$ is

$$
\sum_{R \in \mathcal{R}} g(R)=|\mathcal{R}|-\sum_{R \in \mathcal{R}} q(R)=|\mathcal{R}|-|I|
$$

Our next lemma shows that $g$ is indeed an optimal solution.

Lemma 3. The objective value for $g$ equals the upper bound from the minimal decomposition of $P$ into rectangles. In other words,

$$
|\mathcal{R}|-|I|=\frac{N}{2}-c+k
$$

Proof. Clearly, we may assume $c=1$. We proceed by induction on the objective value $h:=\frac{N}{2}-1+k$. The value $h=1$ is possible only if $P$ is a single rectangle, and in this case $|\mathcal{R}|-|I|=1-0=1$. If $h>0, P$ has at least one concave vertex. Let $a$ be a concave vertex such that no concave vertex is right of $a$. Along the vertical line through $a$ we cut the polygon $P$ into two polygons $P_{1}$ and $P_{2}$. The three possible situations are illustrated in Fig. 9.

For $i \in\{1,2\}$, let $N_{i}$ and $k_{i}$ be the numbers of vertices and holes of the respective polygons, $\mathcal{R}_{i}$ the sets of basic rectangles, $I_{i}$ the sets of intersection points, and let $h_{i}=$ $N_{i} / 2-1+k_{i}$ be the corresponding objective values.

Case (a). We have $N=N_{1}+N_{2}-2$ and $k=k_{1}+k_{2}$, thus $h=h_{1}+h_{2}$. So $h_{1}$ and $h_{2}$ are smaller than $h$ and we can apply the induction hypothesis to $P_{1}$ and $P_{2}$. Let $t$ be the number of intersection points for $P$ that are not in $I_{1} \cup I_{2}$. Clearly, these points lie on the horizontal or on the vertical line through $a$, as indicated in Fig. 10. Let $t_{1}$ be the number of new intersection points on the vertical line, except $a$ itself, and let $t_{2}$ be the number of new intersection points on the horizontal line (including 


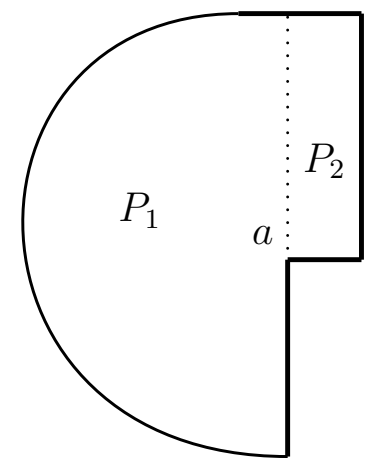

(a)

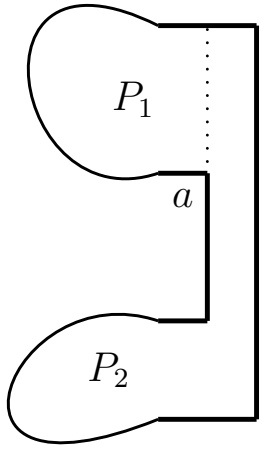

(b)

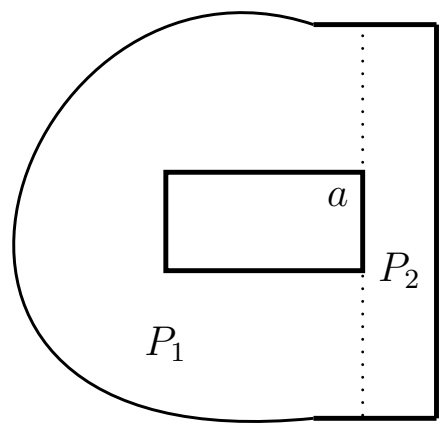

(c)

Figure 9: The possible cuts (dotted lines) in the proof of Lemma 3.

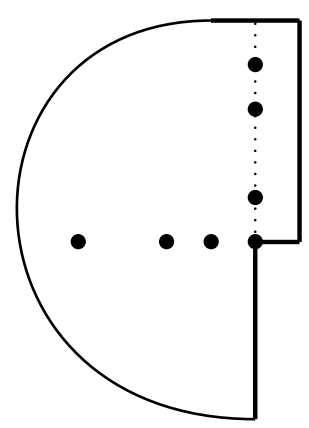

Figure 10: The new intersection points in Case (a).

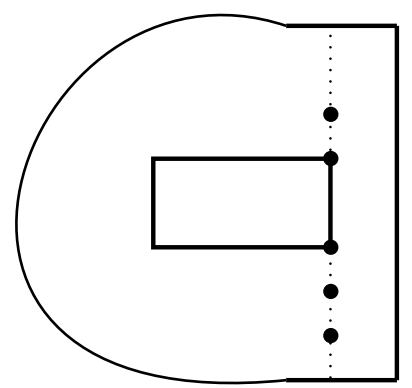

Figure 11: The new intersection points in Case (c).

$a$ ), so $t=t_{1}+t_{2}$. In $P, P_{2}$ is divided into $t_{1}+1$ basic rectangles (which gives $t_{1}$ additional basic rectangles), and exactly $t_{2}$ basic rectangles of $P_{1}$ are cut into two parts. We obtain

$$
|\mathcal{R}|=\left|\mathcal{R}_{1}\right|+\left|\mathcal{R}_{2}\right|+t, \quad|I|=\left|I_{1}\right|+\left|I_{2}\right|+t,
$$

and the claim follows by induction.

Case (b). As in case (a), $h=h_{1}+h_{2}$, and we can apply the induction hypothesis to $P_{1}$ and $P_{2}$. Again, we see that every new intersection point (all on the vertical line through $a$ ) creates a new basic rectangle, and the argument is completed as before.

Case (c). This time we have $N=N_{1}+N_{2}-4$ and $k=k_{1}+k_{2}+1$, but again $h=h_{1}+h_{2}$, so the induction hypothesis applies. Again the $t$ new intersection points lie on the vertical line through $a$, and $P_{2}$ is divided into $t+1$ basic rectangles (see Fig. 11), and this concludes the proof. 


\section{The general case}

In this section we show how the general case can be handled. In the first subsection we describe the solution $g$ in the general case, while the second subsection is devoted to the proof of the main theorem.

\subsection{Definition of the solution}

In order to define the solution as in (1), we have to put an orientation on the chords. To fix such an orientation let $Q=H_{0} \cup V_{0}$ be an independent set of size $|Q|=\alpha(G)$, where $H_{0} \subseteq H$ and $V_{0} \subseteq V$. In addition, let $H_{1}=H \backslash H_{0}$ and $V_{1}=V \backslash V_{0}$. By maximality of $Q$ and Hall's theorem, $H_{1}$ can be matched into $V_{0}$, and $V_{1}$ can be matched into $H_{0}$. Let $M \subseteq\left(H_{0} \times V_{1}\right) \cup\left(H_{1} \times V_{0}\right)$ be such a matching, i.e. $|M|=\left|H_{1}\right|+\left|V_{1}\right|$. Let $Q_{0} \subseteq Q$ be the subset of vertices that are not matched in $M$. In particular, we have $\alpha=\left|Q_{0}\right|+|M|$. We call the elements of $Q_{0}$ isolated chords. Now the vertical and horizontal isolated chords are oriented from top to bottom and from left to right, respectively. For an edge $x y \in M$, we direct every segment of the chords $x$ and $y$ towards the intersection point of $x$ and $y$. Fig. 12 illustrates this for the polygon in Fig. 2 where the underlying matching is $M=\{a 1, b 2, c 3\}$ and the isolated chords are 4 and $d$ (see Fig. 2 for the labeling of the chords). Now we can define $g: \mathcal{R} \rightarrow\{0, \pm 1\}$ by $g(R)=1-q(R)$ as before.

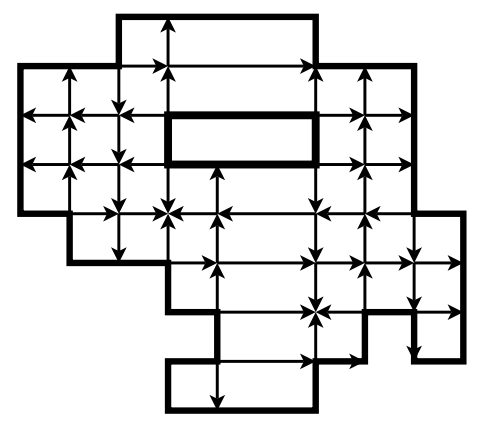

Figure 12: The orientation in the general case.

\subsection{Proof of the main theorem}

When we try to prove the feasibility of $g$ by induction on $|\mathcal{A}|$ as in Lemma 2, a problem arises in the case that $f$ is the endpoint of a horizontal isolated chord as indicated in the left hand side of Fig. 13. Then $f$ is not a source for any of the rectangles $\mathcal{A}_{1}$ and $\mathcal{A}_{2}$. The right hand side of the same figure shows a solution for this problem: we just extend the orientation to the first segment of the boundary immediately following the isolated chord. Of course, we have to do this for every isolated chord. Observe that in Fig. 12 this is done for both isolated chords (which are 4 and $d$ in this example). After this modification the argument for Lemma 2 proves the following general version. 


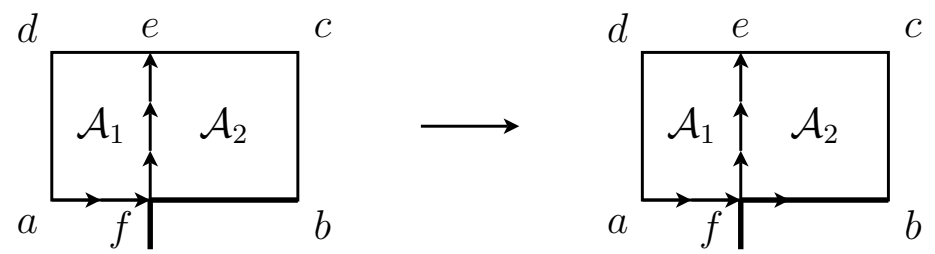

Figure 13: The problem in the induction step for the feasibility of $g$ (and its solution).

Lemma 4. For any essential rectangle $\mathcal{A} \subseteq \mathcal{R}$, we have

$$
\sum_{R \in \mathcal{A}} g(R)=1-q(\mathcal{A})
$$

In particular, $g: \mathcal{R} \rightarrow\{0, \pm 1\}$ defines a feasible solution for $\boldsymbol{R} \boldsymbol{S P}-\boldsymbol{D u a l}$.

Now it remains to prove the optimality of $g$. There are some special intersection points: the points where two chords meet which are matched in $M$. Clearly, these are never a source for any incident basic rectangle. But each of the remaining intersection points is a source for precisely one basic rectangle. So we obtain the objective value

$$
\sum_{R \in \mathcal{R}} g(R)=|\mathcal{R}|-|I|+|M|
$$

Lemma 5. We have

$$
|\mathcal{R}|-|I|+|M|=\frac{N}{2}-c+k-\alpha .
$$

Proof. As in the proof of Lemma 3 we may assume $c=1$. We proceed by induction on $\alpha$. The case $\alpha=0$ was treated in Section 3. So assume $\alpha>0$, let $Q$ be some independent set of chords of size $|Q|=\alpha$, and choose any chord $a b \in Q$ (w.l.o.g. horizontal). Now we cut the polygon $P$ along the chord $a b$. We have to distinguish two different cases: either the cut divides $P$ into two polygons $P_{1}$ and $P_{2}$, or $P$ stays connected, but the number of holes decreases by 1 (see Fig. 14 and 17).

Case 1 (Fig. 14). For $i \in\{1,2\}$, denote the parameters of $P_{i}$ by $N_{i}, k_{i}$ and $\alpha_{i}$. Similarly, the corresponding sets of basic rectangles and intersection points are denoted by $\mathcal{R}_{i}$ and $I_{i}$, respectively. Observe that $P_{i}$ inherits a maximum independent set $Q_{i}$ and a corresponding Matching $M_{i}$ from $P: Q_{i}$ is the set of chords in $P$ that are also chords in $P_{i}$, and $M_{i}$ is the set of elements $x y \in M$ such that $x$ and $y$ are both in $Q_{i}$. We have

$$
N=N_{1}+N_{2}, \quad k=k_{1}+k_{2}, \quad \alpha=\alpha_{1}+\alpha_{2}+1 .
$$

Hence, by induction, it is sufficient to prove that

$$
|\mathcal{R}|-|I|+|M|=\left(\left|\mathcal{R}_{1}\right|-\left|I_{1}\right|+\left|M_{1}\right|\right)+\left(\left|\mathcal{R}_{2}\right|-\left|I_{2}\right|+\left|M_{2}\right|\right) .
$$



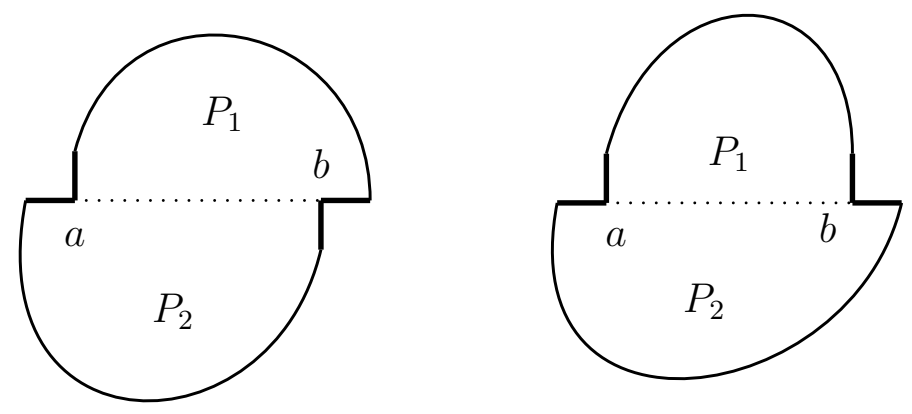

Figure 14: A cut dividing $P$ into two parts (Case 1).

Let $T$ be the set of intersection points on the chord $a b$. This set splits into three subsets. For $i \in\{1,2\}$, we put

$$
\begin{array}{r}
T_{i}:=\left\{u \in T: u \text { is a vertex of an element of } \mathcal{R}_{i},\right. \\
\text { but not of an element of } \left.\mathcal{R}_{3-i} .\right\},
\end{array}
$$

and in addition $T_{3}=T \backslash\left(T_{1} \cup T_{2}\right)$. Observe that $T_{3}$ is the set of points where the chord $a b$ meets other chords. For instance, in Fig. 15, we have

$$
T_{1}=\left\{a_{1}, a_{3}\right\}, \quad T_{2}=\left\{a_{2}, a_{4}\right\}, \quad T_{3}=\left\{a_{5}, a_{6}\right\} .
$$

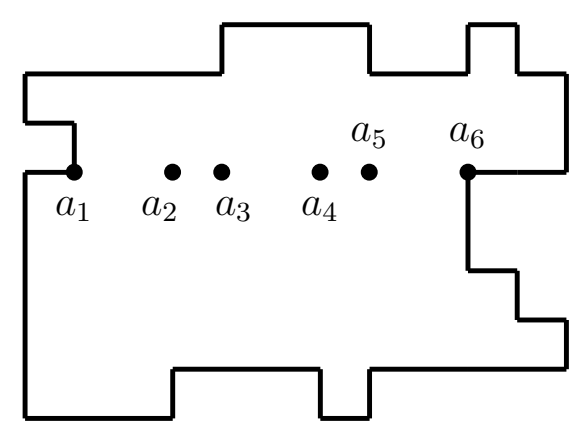

Figure 15: The intersection points on the cutting chord.

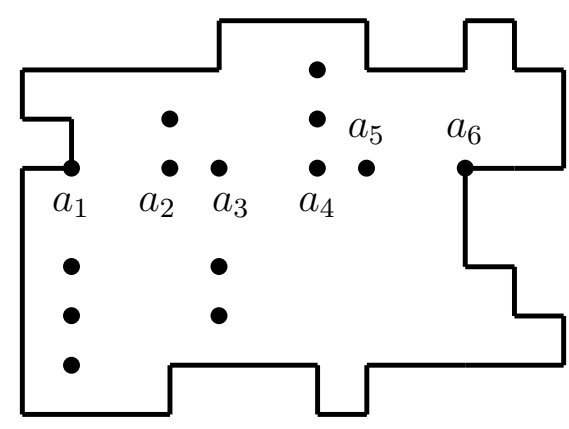

Figure 16: The additional intersection points.

Next we define a function $\phi: T_{1} \cup T_{2} \rightarrow \mathbb{N}$. For $u$ in $T_{1}$, let $\phi(u)$ be the number of intersection points for $P$ on the vertical line through $u$ lying below $u$. Similarly, for $u$ in $T_{2}$, let $\phi(u)$ be the number of intersection points for $P$ on the vertical line through $u$ lying above $u$. In both cases, the point $u$ itself is included. In the example from Fig. 15, we have $\phi\left(a_{1}\right)=4, \phi\left(a_{2}\right)=2$ and $\phi\left(a_{3}\right)=\phi\left(a_{4}\right)=3$ (see Fig. 16). The function $\phi$ counts the intersection points of $P$ that are not intersection points of $P_{1}$ or $P_{2}$ :

$$
|I|=\left|I_{1}\right|+\left|I_{2}\right|+\sum_{c \in T_{1} \cup T_{2}} \phi(c)+\left|T_{3}\right| .
$$


On the other hand, $\phi$ can be used to count the additional basic rectangles. The vertical line through $u \in T_{1}$ divides $\phi(u)$ basic rectangles of $P_{2}$ into two parts, and the vertical line through $u \in T_{2}$ divides $\phi(u)$ basic rectangles of $P_{1}$ into two parts, hence

$$
|\mathcal{R}|=\left|\mathcal{R}_{1}\right|+\left|\mathcal{R}_{2}\right|+\sum_{u \in T_{1} \cup T_{2}} \phi(u)
$$

The matching $M$ can be written as a disjoint union $M=M_{1} \cup M_{2} \cup M_{3}$ as follows. For $i \in\{1,2\}, M_{i}$ is the set of edges $x y \in M$ such that $x \in H, y \in V$, the chord $y$ does not intersect $a b$, and both vertices of $y$ are in $P_{i}$. This is consistent with the above description of the matchings $M_{1}$ and $M_{2}$. The remaining matching edges $x y \in M$ such that $x \in H, y \in V$, and the chord $y$ intersects $a b$, are collected in $M_{3}$. Clearly, for every such matching edge $x y \in M_{3}$, the vertical chord $y$ intersects the chord $a b$ in some point from $T_{3}$. On the other hand, for every point $a^{\prime} \in T_{3}$, the chord $y$ that intersects $a b$ in $a^{\prime}$ does not belong to our maximal independent set $Q$, so it is matched in $M$. Consequently, there is a one-to-one correspondence between $M_{3}$ and $T_{3}$, and we obtain

$$
|M|=\left|M_{1}\right|+\left|M_{2}\right|+\left|T_{3}\right|
$$

Putting together equations (3), (4) and (5), we obtain

$$
\begin{aligned}
|\mathcal{R}|-|I|+|M|= & \left(\left|\mathcal{R}_{1}\right|+\left|\mathcal{R}_{2}\right|+\sum_{u \in T_{1} \cup T_{2}} \phi(u)\right) \\
& -\left(\left|I_{1}\right|+\left|I_{2}\right|+\sum_{u \in T_{1} \cup T_{2}} \phi(u)+\left|T_{3}\right|\right)+\left(\left|M_{1}\right|+\left|M_{2}\right|+\left|T_{3}\right|\right) .
\end{aligned}
$$

This is (2), and thus concludes the proof in this case.

Case 2 (Fig. 17). Essentially the proof is the same as in Case 1. For the parameters of $P^{\prime}$, we obtain

$$
N^{\prime}=N, \quad k^{\prime}=k-1, \quad \alpha^{\prime}=\alpha-1 .
$$

So induction applies to $P^{\prime}$, and we have to show that

$$
|\mathcal{R}|-|I|+|M|=\left|\mathcal{R}^{\prime}\right|-\left|I^{\prime}\right|+\left|M^{\prime}\right|
$$

As before, $T$ is the set of intersection points on the chord $a b$. In analogy to Case $1, T_{1}$ is the set of $u \in T$ such that $u$ sees a concave vertex when it looks upwards, but $u$ does not see a concave vertex when it looks downwards. Similarly, $T_{2}$ is the set of $u \in T$ such that $u$ sees a concave vertex when it looks downwards, but $u$ does not see a concave vertex when it looks upwards, and finally $T_{3}=T \backslash\left(T_{1} \cup T_{2}\right)$, the set of points where $a b$ meets other chords. For $u \in T_{1}$, let $\phi(u)$ be the number of intersection points on the vertical line 

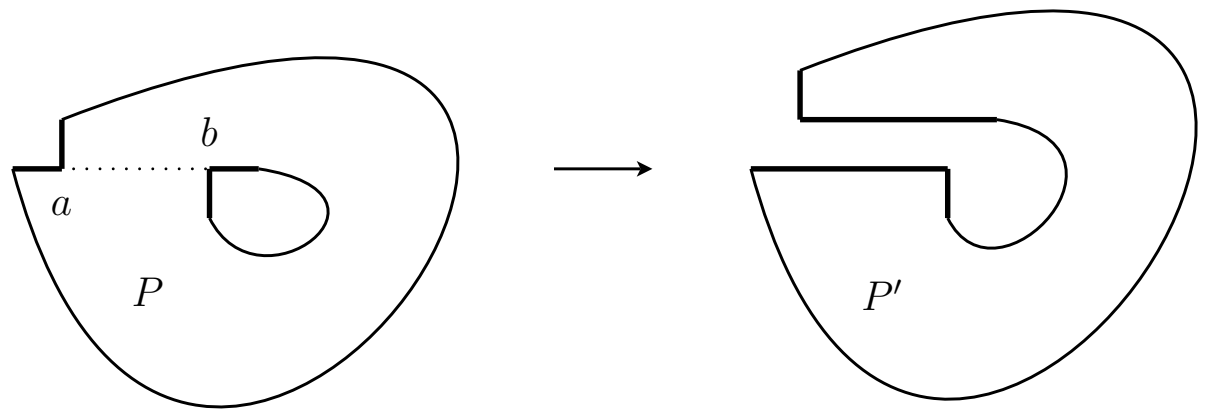

Figure 17: A cut that kills a hole (Case 2).

through $u$ lying below $u$, and for $u \in T_{2}$, let $\phi(u)$ be the number of intersection points on the vertical line through $u$ lying above $u$ (in both cases we include $u$ itself). By the same counting arguments as in Case 1 we obtain

$$
\begin{aligned}
|I| & =\left|I^{\prime}\right|+\sum_{u \in T_{1} \cup T_{2}} \phi(u)+\left|T_{3}\right|, \\
|\mathcal{R}| & =\left|\mathcal{R}^{\prime}\right|+\sum_{u \in T_{1} \cup T_{2}} \phi(u), \\
|M| & =\left|M^{\prime}\right|-\left|T_{3}\right| .
\end{aligned}
$$

These equations imply (6), and this concludes the proof.

As a consequence of Lemmas 4 and 5, we obtain that $g$ solves RSP-Dual.

Theorem 2. The function $g: \mathcal{R} \rightarrow\{0, \pm 1\}$ with $g(A)=1-q(A)$ defines an optimal solution for $\boldsymbol{R S P}$-Dual.

Corollary 1. There is no integrality gap in the rectangle segmentation problem for binary input matrices.

\section{References}

[1] K. Engel. Optimal matrix-segmentation by rectangles. Discr. Appl. Math., 2009. in press. doi:10.1016/j.dam.2008.12.008.

[2] L. Ferrari, P.V. Sankar, and J. Sklansky. Minimal rectangular partitions of digitized blobs. Computer Vision, Graphics and Image Processing, 28:58-71, 1984.

[3] W. Lipski, E. Lodi, F. Luccio, C. Mugnai, and L. Pagli. On two dimensional data organization II. Fund. Informaticae, 2:245-260, 1979.

[4] T. Ohtsuki. Minimum dissection of rectilinear regions. In Proceedings of IEEE Symposium on Circuits and Systems, pages 1210-1213, 1982. 